\title{
First-principles study of phase transitions inKNO3
}

\section{Citation}

Lu, H. M., and J. R. Hardy. 1991. "First-Principles Study of Phase Transitions inKN03." Physical Review B 44 (14) (October 1): 7215-7224. doi:10.1103/physrevb.44.7215.

\section{Published Version}

doi:10.1103/PhysRevB.44.7215

\section{Permanent link}

http://nrs.harvard.edu/urn-3:HUL.InstRepos:32430087

\section{Terms of Use}

This article was downloaded from Harvard University's DASH repository, and is made available under the terms and conditions applicable to Other Posted Material, as set forth at http:// nrs.harvard.edu/urn-3:HUL.InstRepos:dash.current.terms-of-use\#LAA

\section{Share Your Story}

The Harvard community has made this article openly available.

Please share how this access benefits you. Submit a story.

\section{Accessibility}




\title{
First-principles study of phase transitions in $\mathrm{KNO}_{3}$
}

\author{
H. M. Lu and J. R. Hardy \\ Department of Physics and Center for Electro-Optics, University of Nebraska-Lincoln, Lincoln, Nebraska 68588-0111
}

(Received 21 December 1990; revised manuscript received 20 May 1991)

\begin{abstract}
We report a first-principles simulation study of phase transitions in $\mathrm{KNO}_{3}$, using our recently developed method for treating ionic molecular solids. With the interionic potentials calculated from $a b$ initio electron charge densities of the ions, our structural static relaxation gave close fits to both the normal room-temperature ( $\alpha$-phase) and the ferroelectric ( $\gamma$-phase) structures. Our supercell moleculardynamics calculations closely simulated the transitions from the $\alpha$ phase and $\gamma$ phase to the hightemperature disordered $\beta$ phase, and successfully reproduced the abnormally large c-axis thermal expansion observed in experiment. Both transitions were found to be initiated by the rotations of the nitrate ions about their triad axes parallel to the c axis. Our lattice-dynamics calculations in the spirit of the quasiharmonic approximation revealed that these rotations are intimately connected to the large c-axis thermal expansion, and both of them derive from the strong anharmonicity in the system.
\end{abstract}

\section{INTRODUCTION}

Recently we have developed a first-principles approach to simulation studies of ionic molecular crystals and have successfully reproduced the phase transitions in $\mathrm{K}_{2} \mathrm{SeO}_{4}$ crystal. $^{1,2}$ To account consistently for the effect of electron covalency within the molecular ions, the method starts from $a b$ initio quantum-chemistry calculations for the whole molecular ion to obtain the true electron charge distribution. Then, by treating the predominantly covalent intramolecular interactions with $a b$ initio Taylor expansions of the molecular ion's energy, and by treating the largely ionic intermolecular interactions using the Gordon-Kim electron-gas model ${ }^{3}$ known to be effective for purely ionic crystals, ${ }^{4-10}$ the method provides an accurate description for the crystal potential-energy surfaces. Structural relaxation, molecular-dynamics simulation, and lattice-dynamics calculations, based on these potential surfaces, allow one to study delicately balanced competing instabilities and reveal their origins at the microscopic level.

In the present paper, we report our studies on potassium nitrate $\left(\mathrm{KNO}_{3}\right)$ which exhibits an interesting phase sequence at atmospheric pressure. ${ }^{11,12}$ When heated from the room-temperature orthorhombic (space group Pmcn) structure ( $\alpha$ phase), it undergoes a transition at $128^{\circ} \mathrm{C}$ to a trigonal structure ( $\beta$ phase), which is dynamically disordered with the nearly free rotations of the $\mathrm{NO}_{3}{ }^{-}$ ions. On cooling from the $\beta$ phase, it does not transform directly back to the $\alpha$ phase, but instead passes into another trigonal phase $(\gamma)$ between 124 and $110^{\circ} \mathrm{C}$, which is ferroelectric with the spontaneous polarization along the c axis. ${ }^{13}$ It was found that the temperature range over which $\gamma \mathrm{KNO}_{3}$ exists depends on the previous treatment of the sample and the cooling rate, and it can persist down to room temperature. ${ }^{11,12}$ These peculiar properties of $\mathrm{KNO}_{3}$ have stimulated several structur$\mathrm{al}^{12,13}$ as well as dynamical ${ }^{14-16}$ experimental studies. There is not, however, a theoretical study at the microscopic level.
In this work we describe first-principles studies of phase transitions in $\mathrm{KNO}_{3}$, using our new method outlined above for ionic molecular solids. These studies provide a further test for the new method and in particular its description for the intramolecular and intermolecular interactions involving the nitrate ion, which is less symmetric compared to the selenate ion in $\mathrm{K}_{2} \mathrm{SeO}_{4}$ for which our theory was very successful. ${ }^{2}$ Such a description is very valuable in that it is truly ab initio (parameter free) and therefore can be used to predict properties of known materials or novel materials, for example, a mixture of $\mathrm{KNO}_{3}$ and $\mathrm{NaNO}_{3}$. In addition, these studies of the phase transitions in $\mathrm{KNO}_{3}$ may provide some general understanding of the rotational disordering transitions that exist in many ionic molecular solids, e.g., $\mathrm{NaNO}_{3}$, and possibly $\mathrm{CaCO}_{3}$.

Following the general approach outlined above for ionic molecular crystals we performed quantum chemistry calculation for the $\mathrm{NO}_{3}{ }^{-}$ion, and calculated the interionic potentials for $\mathrm{KNO}_{3}$. Using these potentials we then performed static structural relaxations for the roomtemperature $\alpha$ phase and the ferroelectric $\gamma$ phase. The relaxed theoretical structures were found to be in close agreement with experiment, indicating that the interionic potentials were reliable. Then supercell molecular dynamics calculations were performed to simulate directly the order-disorder $\alpha \rightarrow \beta$ and $\gamma \rightarrow \beta$ transitions. It was found that both transitions were initiated by rotations of $\mathrm{NO}_{3}{ }^{-}$ions about their triad axes parallel to $\mathrm{c}$ and were accompanied by abnormally large thermal expansions in the $\mathrm{c}$ direction. In order to investigate the connection between these rotations of the $\mathrm{NO}_{3}{ }^{-}$ions and the large caxis expansion, we performed lattice-dynamics calculations for different temperatures in the spirit of the quasiharmonic approximation. We found that the frequencies of the normal modes that involve rotations of the nitrate ions about their triad axes fall drastically when the $c$ axis expands as the temperature approaches the transition temperature. Therefore, the abnormally large c-axis expansion is closely related to the order- 
disorder phase transitions in $\mathrm{KNO}_{3}$. This important character of the rotational motion of the nitrate ions was also evident from a temperature-dependent Fouriertransform analysis of such motions in our moleculardynamics simulation.

This paper is organized as follows: In Sec. II, we present the calculation for obtaining the intra- and intermolecular potentials. Section III gives the results of static structural relaxations for both the room-temperature Pmcn and the ferroelectric $R 3 m$ structures. In Sec. IV, we present the molecular-dynamics calculations which directly simulate the phase transitions. Section $V$ gives the results of quasiharmonic lattice dynamics calculations and the Fourier analyses of the rotational motions of the nitrate ions in the molecular dynamics simulation. Section VI concludes the paper.

\section{CALCULATIONS OF THE $a b$ initio INTER-AND INTRAMOLECULAR POTENTIALS}

A full description of the procedure for obtaining interionic potentials from $a b$ initio quantum chemistry calculations has been given in Ref. 2 , therefore here we only outline the steps. We first performed a quantum chemistry structural optimization for a nitrate ion, which searches for the atomic configuration that gives the lowest self-consistent Hartree-Fock energy. Such an optimization is necessary to provide an equilibrium structure for a harmonic expansion of the total energy of the molecular ion as a function of the bond lengths, bond angles, etc. The optimized structure of $\mathrm{NO}_{3}{ }^{-}$is planar with the $\mathrm{N}-\mathrm{O}$ bond length being $1.23 \AA$, very close to the experimental value of $1.24 \AA$ in the $\alpha$ phase, ${ }^{12}$ indicating that a harmonic expansion should provide a close description of the intramolecular interactions. $A b$ initio force constants for the optimized structure of the $\mathrm{NO}_{3}{ }^{-}$ ion were then calculated to be used for the harmonic expansion.

Next we calculated the electron charge density distribution for the $\mathrm{NO}_{3}{ }^{-}$ion at the optimized structure. This charge density distribution was then decomposed ${ }^{2}$ into approximate charge densities for the individual nitrogen and oxygen atoms. Using these charge densities and the free ion charge density for the $\mathrm{K}^{+}$ion, ${ }^{17}$ we then computed the short-range pair potentials between these ions according to the Gordon-Kim electron gas model. ${ }^{3}$ For the long-range Columb interactions, the charges on the ions were +1 for $\mathrm{K}^{+}, 0.9917$ for $\mathrm{N}$, and -0.6639 for $\mathrm{O}$, where the fractional charges were obtained from the computed charge density of $\mathrm{NO}_{3}{ }^{-}$by a Mulliken population analysis. ${ }^{18}$

The quantum chemistry calculations were performed using the GAUSSIAN86 program. ${ }^{19}$ For both the oxygen and nitrogen atoms the standard 6-31G basis sets were used and the single first polarization functions were added to the basis sets.

Our quantum chemistry calculations were made for an isolated $\mathrm{NO}_{3}{ }^{-}$ion and thus did not include a background crystal field. Such a background crystal field will definitely affect the electron charge density distribution of $\mathrm{NO}_{3}{ }^{-}$, but these effects may well be unimportant for the following reason. By regarding the atoms as point charges with the values cited above, we calculated the electric fields at the atomic sites in the experimental structure. We found that while the dominating fields are those on the oxygen atoms and lie in the $\mathrm{NO}_{3}^{-}$planes, over $90 \%$ of the contribution to these fields is from the other atoms in the same $\mathrm{NO}_{3}^{-}$group, rather than from the rest of the lattice.

This was true for both the room-temperature Pmcn structure and the ferroelectric $R 3 \mathrm{~m}$ structure. Therefore, by performing the quantum chemistry calculations for the whole $\mathrm{NO}_{3}{ }^{-}$ion, we have already included most of the effects of the actual crystal field. Although the rest of the crystal fields may affect, to some degree, the shapes of the tails of the electron charge distributions on the outskirts of the $\mathrm{NO}_{3}{ }^{-}$, they are relatively unimportant in determining the electron charge distribution within the $\mathrm{NO}_{3}{ }^{-}$ion which is the most crucial for both intra- and intermolecular interactions.

\section{STATIC Pmcn and $R 3 m$ STRUCTURES OF $\mathrm{KNO}_{3}$}

With the intra- and intermolecular potentials determined, we then performed static relaxations for the room-temperature $P m c n$ and the ferroelectric $R 3 m$ structures of $\mathrm{KNO}_{3}$. These relaxations gave the crystal structures that are at the extreme of the theoretical potentialenergy surfaces, where the forces on the ions and the stresses are zero. Comparisons of the theoretical structures with the experimental data provide sensitive tests of the validity of the theoretical potential energy surfaces for the system. Our relaxation was performed for infinite lattices by applying periodic boundary conditions, and followed a Newton-Raphson algorithm. The standard technique of the Ewald sum was used to calculate the lattice energy and forces, etc.

First we considered the $\alpha$ phase of $\mathrm{KNO}_{3}$. The space group was Pmcn and it has four formula units per unit cell. Figure 1 shows the projections of the experimental ion positions at $25^{\circ} \mathrm{C}$ (Ref. 12) with the structural parameters given in Table I. We started our static relaxation from this experimental structure under the constraint of Pmcn symmetry, i.e., only the parameters listed in Table I were allowed to vary. The system relaxed easily to a structure with zero stresses and zero forces on the ions. The parameters for this theoretical structure are listed in Table I and the projections of ions are given in Fig. 2.

The lattice constants of the theoretical structure are shorter than the experimental values by $2.9 \%$ for $a, 4.1 \%$ for $b$, and $8.8 \%$ for $c$. The shortening of the $\mathbf{a}$ and $\mathbf{b}$ axes should be considered reasonable, since this type of shortening is a rather general feature for large-unit-cell simulations using Gordon-Kim potentials. While the shortening of the c axis is obviously too large, it is to be expected from the relatively loose packing of the ions along the $c$ direction (owing to the fact that the $\mathrm{NO}_{3}{ }^{-}$ions lie in the $\mathbf{a}, \mathbf{b}$ plane) since this static relaxation omits the kinetic energy present at finite temperatures. Usually temperature does not increase the lattice constants anomalously, but for the present structure with fairly weak interactions in the c direction, due to the loose packing, temperature 

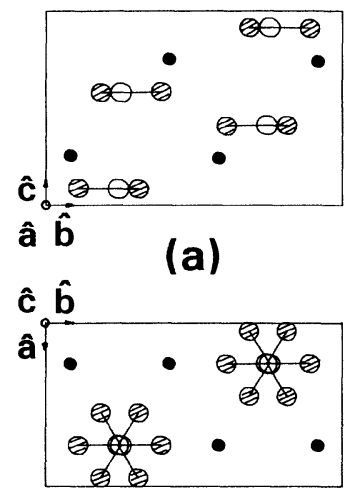

(b)

FIG. 1. Projections of the experimentally determined average structure of $\mathrm{KNO}_{3}$ in the Pmcn phase. The unit vectors indicate the directions of the lattice vectors. A unit vector pointing out of the page is represented by a circle. The $\mathbf{N}$ atoms are represented by open circles, $\mathrm{K}$ atoms by dark circles, and $\mathrm{O}$ atoms by shaded circles. Bonds (straight lines) connect each $\mathbf{N}$ and the nearest three $\mathrm{O}$ atoms.

effects could be important. In fact, as will be seen subsequently, when we performed supercell molecular dynamics calculations at finite temperature, the $c$ axis increased rapidly as the temperature was raised. At $T=300 \mathrm{~K}$, at which the experimental data were taken, ${ }^{12}$ the c-axis shortening decreased to $5.2 \%$. Although it is still larger than the $a-$ and $b$-axes shortenings of, $2.3 \%$ and $3.5 \%$, respectively, at this temperature, the differences between them are much smaller. (The abnormally large thermal expansion in the c direction has been seen in experiment, as will be discussed in Sec. IV.)

Apart from this shortening of the lattice constants, the agreement between theory and experiment is very good, as can be seen in Table I. In fact, if the experimental lattice constants were used for both structures, the differences between the ion positions were all less than 0.1 bohr.

TABLE I. Experimental ${ }^{12}$ and theoretical structural parameters for the Pmcn structure of $\mathrm{KNO}_{3}$. The lattice constants are given in Angstroms.

\begin{tabular}{lrr}
\hline Parameters & Experiment & Theory \\
\hline$a$ & 5.4119 & 5.2555 \\
$b$ & 9.1567 & 8.7826 \\
$c$ & 6.4213 & 5.8537 \\
$y / b$ of $\mathrm{K}$ & 0.4166 & 0.4155 \\
$z / c$ of $\mathrm{K}$ & 0.7568 & 0.7562 \\
$y / b$ of $\mathrm{N}$ & 0.7548 & 0.7555 \\
$z / c$ of $\mathrm{N}$ & -0.0848 & 0.0867 \\
$y / b$ of $\mathrm{O}(1)$ & 0.8902 & 0.8946 \\
$z / c$ of $\mathrm{O}(1)$ & -0.0893 & 0.0893 \\
$x / a$ of $\mathrm{O}(2)$ & 0.4492 & 0.4512 \\
$y / b$ of $\mathrm{O}(2)$ & 0.6866 & 0.6859 \\
$z / c$ of $\mathrm{O}(2)$ & -0.0849 & 0.0862 \\
\hline \hline
\end{tabular}

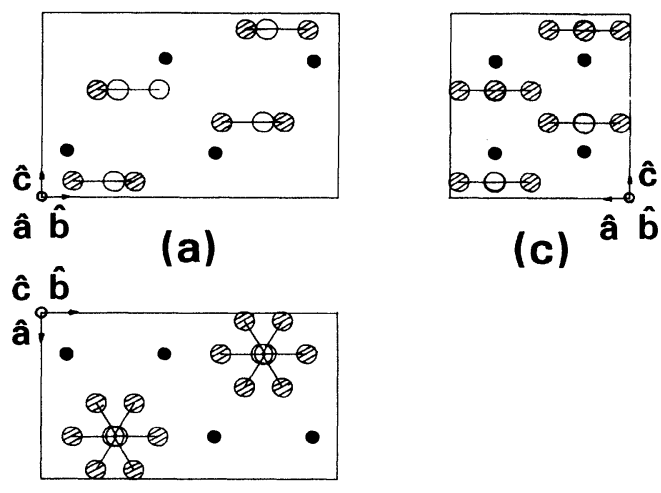

(b)

FIG. 2. Projections of the theoretical Pmcn structure obtained by static relaxation with $P m c n$ constraint.

We also performed a static relaxation for the $\alpha$ phase of $\mathrm{KNO}_{3}$ without the Pmcn symmetry constraints. The resultant structure is exactly that given by relaxation with the Pmcn constraints, i.e., that given in Fig. 2 and Table I.

Next we performed a similar static relaxation for the ferroelectric $\gamma$-phase of $\mathrm{KNO}_{3}$. Figure 3 gives the projections of the experimental ion positions. ${ }^{11}$ The symmetry of the structure is $R 3 m$ with three formula units per unit
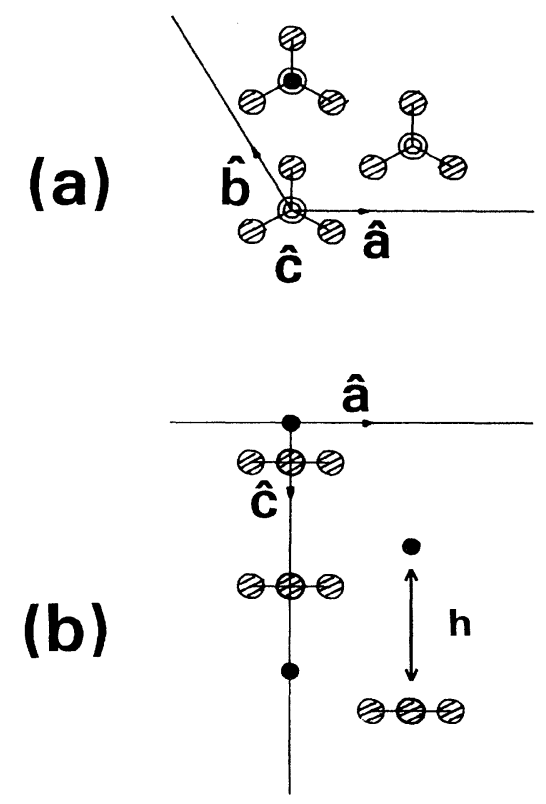

FIG. 3. Projections of the experimentally determined average structure of $\mathrm{KNO}_{3}$ in the ferroelectric $R 3 \mathrm{~m}$ phase. The parameter $h$ gives the distance between a nitrate ion and a potassium ion just above. For this structure $h=0.44 c$, which is smaller than $0.5 c$, thereby giving rise to a spontaneous polarization in the c direction. 
TABLE II. Experimental ${ }^{11}$ and theoretical structural parameters for the ferroelectric $R 3 m$ (hexagonal) structure of $\mathrm{KNO}_{3}$. The lattice constants are given in Angstroms.

\begin{tabular}{lcc}
\hline \hline Parameters & Experiment & Theory \\
\hline$a(=b)$ & 5.482 & 5.283 \\
$c$ & 9.144 & 8.146 \\
$x / a$ of $\mathrm{O}$ & 0.11 & 0.133 \\
$z / c$ of $\mathrm{O}$ & 0.44 & 0.433 \\
$z / c$ of $\mathrm{N}$ & 0.44 & 0.435 \\
\hline \hline
\end{tabular}

cell. The $\mathrm{NO}_{3}{ }^{-}$groups lie in the a,b plane with the potassium ions being exactly above and under the nitrogen ion. However, the $\mathrm{NO}_{3}{ }^{-}$group is not exactly halfway between the $\mathrm{K}^{+}$ions, thus giving rise to a spontaneous polarization in the c direction, which is reversible by shifts of $\mathrm{NO}_{3}{ }^{-}$groups also in the c direction and with appropriate rotations about their triad axes. ${ }^{11}$ The structural parameters for the experimental structure are given in Table II.

As we did for the Pmcn structure, we first performed static relaxation with the $R 3 m$ symmetry constants; i.e., only the structural parameters listed in Table II were allowed to vary. The relaxed structure is plotted in Fig. 4, with the parameters given in Table II. As was the case for the Pmcn structure, the reduced basis positions in the theoretical structure are very close to the experimental values and again the lattice constants are too short: $3.6 \%$ for $a(=b)$ and $11 \%$ for $c$. This rather significant shortening of $c$ axis has the same origin, i.e., the loose packing in the $R 3 \mathrm{~m}$ structure along the c direction, and is seen to improve markedly when temperature effects are included via molecular dynamics simulations.
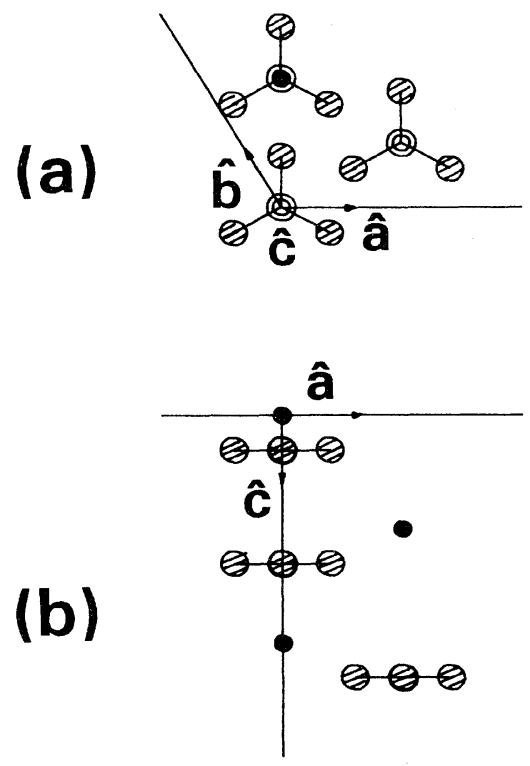

FIG. 4. Projections of the theoretical $R 3 m$ structure obtained by static relaxation with $R 3 m$ constraint.
In an x-ray study of the $R 3 m$ structure, Nimmo and Lucas $^{12}$ concluded that the nitrogen atom is not in the plane formed by the three oxygen atoms, but displaced out of it by about half a Bohr, which is contrary to the earlier experimental study ${ }^{11}$ (Table II). In our theoretical static structure, the $\mathrm{NO}_{3}{ }^{-}$groups are also planar, although this could be changed when temperature effects are included.

The experimental value ${ }^{13}$ for the spontaneous polarization is $8 \mu \mathrm{C} / \mathrm{cm}^{2}$. However, a calculation ${ }^{13}$ using the experimental atomic positions gave the polarization as 11 $\mu \mathrm{C} / \mathrm{cm}^{2}$. This difference was attributed to the incomplete alignment of ferroelectric domains in the sample. ${ }^{13}$ The polarization for our theoretical structure is $13 \mu \mathrm{C} / \mathrm{cm}^{2}$. We note that this over large value is mainly the result of the shortening of the lattice constants, giving a smaller volume for the unit cell in the theoretical structure.

We also performed static relaxation for the $R 3 m$ structure without the $R 3 m$ symmetry constraints. The same structure was obtained as the relaxation with the $R 3 \mathrm{~m}$ symmetry constraints.

The potential energy in the theoretical $R 3 m$ structure is $88 \mathrm{meV}$ per formula unit higher than the theoretical Pmcn structure. As will be shown in the following sections, the $R 3 \mathrm{~m}$ structure is stable against molecular dynamics relaxation and does not exhibit any imaginary phonon branches. Therefore, the $R 3 m$ structure is a metastable state with significant stability, which is consistent with the experimental fact that the transition temperature from the $\gamma$ phase to the $\alpha$ phase depends on the cooling speed and that the $\gamma$ phase can actually be retained down to room temperature by fast cooling. ${ }^{11}$

Overall, our theoretical potential-energy surfaces gave reasonable fits to the experimental structures. The biggest differences are for the lattice constants. The shortening of the $\mathbf{a}$ and $\mathbf{b}$ axes should be considered normal, as far as simulations using Gordon-Kim pair potentials are concerned, as was pointed out earlier. While the neglect of the thermal energy effects in the theoretical structures is mainly responsible for the abnormally large shortening of the $\mathrm{c}$ axis, the planar structure of the $\mathrm{NO}_{3}{ }^{-}$ions may impair the accuracy of our method for the calculation of the intermolecular potentials. As was outlined in Sec. II, in order to calculate the intermolecular pair potentials, e.g., the $\mathrm{K}-\mathrm{O}$ and $\mathrm{K}-\mathrm{N}$ pair potentials, we had to separate the exact total electron charge density for the whole $\mathrm{NO}_{3}{ }^{-}$ion, obtained from quantum chemistry calculations, into approximate spherical charge densities for the individual atoms. While such a separation seems to work well for highly symmetric molecular ions, e.g., for $\mathrm{SeO}_{4}{ }^{2-}$ in our study ${ }^{1,2}$ of $\mathrm{K}_{2} \mathrm{SeO}_{4}$ and for $\mathrm{SO}_{4}{ }^{2-}$ in our study ${ }^{20}$ of $\mathrm{K}_{2} \mathrm{SO}_{4}$, which are perfect tetrahedra in their free form, it could have shortcomings for the planar $\mathrm{NO}_{3}{ }^{-}$ion. Specifically, we found that in the direction perpendicular to the $\mathrm{NO}_{3}{ }^{-}$plane, the total charge density obtained by a superposition of the approximate spherical charge densities for the individual atoms is noticeably smaller than the exact total charge density of $\mathrm{NO}_{3}{ }^{-}$. Thus, the pair potentials calculated using the spherical charge densities may not provide enough repulsive force in the c direction: hence, the shortening of the c axes. A natural way 
to avoid this approximation is to employ molecule-ion and molecule-molecule potentials calculated directly from the exact total charge densities of the molecular ions. However, such potentials would be extremely complicated to calculate and even harder to employ in lattice energy calculations, and more importantly, do not seem to be necessary for our understanding of the origins of the order-disorder phase transitions in $\mathrm{KNO}_{3}$. In particular, our present potential-energy surfaces describe successfully the abnormally large thermal expansion in the c direction, which is central to the phase transitions in $\mathrm{KNO}_{3}$.

\section{DYNAMICS SIMULATION OF THE TRANSITIONS}

\section{A. The $\alpha \rightarrow \beta$ transition}

In our simulation we used a supercell with 540 ions formed by tripling the lower temperature Pmcn cell in all three directions. We started from our theoretical Pmcn structure obtained in Sec. III and heated the sample in stages, $20 \mathrm{~K}$ each time. At each stage the average of the ion positions, the energy, etc., were taken over 2 ps using a 0.005 ps molecular-dynamics time step.

Figure 5 shows the derivative of the average energies with respect to temperature, $d U / d T$, i.e., the specific heat. A clear peak appears at about $440 \mathrm{~K}$, signaling a transition. By examining the average ion positions in the structures below and above $440 \mathrm{~K}$, we found that the transition was associated with the rotations of the $\mathrm{NO}_{3}{ }^{-}$ groups mainly about their triad axes. A convenient and sensitive quantity for monitoring the rotations of the $\mathrm{NO}_{3}{ }^{-}$, and therefore the transition, is the deviation of the orientations of the $\mathrm{N}-\mathrm{O}$ bonds at each temperature from their orientations in the lower temperature Pmcn structure. Thus, we calculated the rms value of these deviations $(R)$ as an auxiliary order parameter, and the result is shown in Fig. 6 as a function of temperature.

As can be seen, the parameter $R$ first increases rather slowly as the temperature is raised, and then jumps to very large values at $\sim 440 \mathrm{~K}$. This abrupt increase of $R$

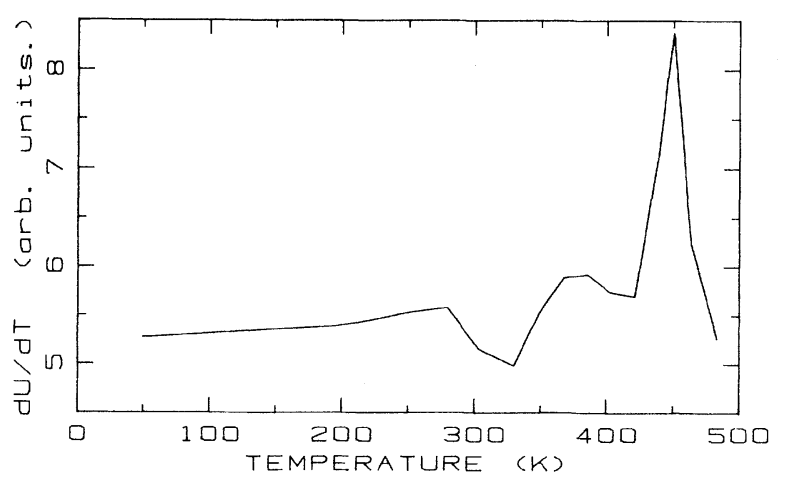

FIG. 5. Specific heat (in arbitrary units) as a function of temperature obtained from the supercell molecular dynamics simulation of the $\alpha \rightarrow \beta$ transition.

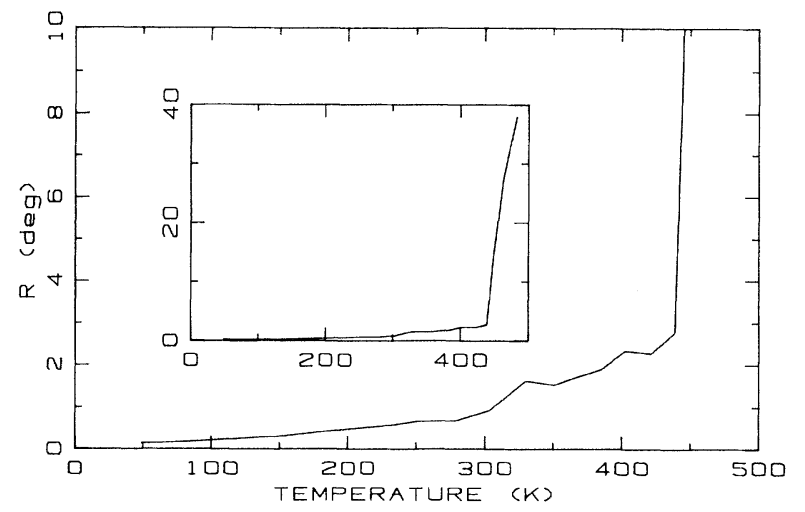

FIG. 6. Auxiliary order parameter $R$ as a function of temperature.

indicates the abrupt onset of large rotations of the $\mathrm{NO}_{3}{ }^{-}$ groups and thus of the transition.

In Fig. 7 we plot the projections of the ion positions parallel to the c direction in the average structures below (a) and above (b) $440 \mathrm{~K}$. In these plots the lines connect

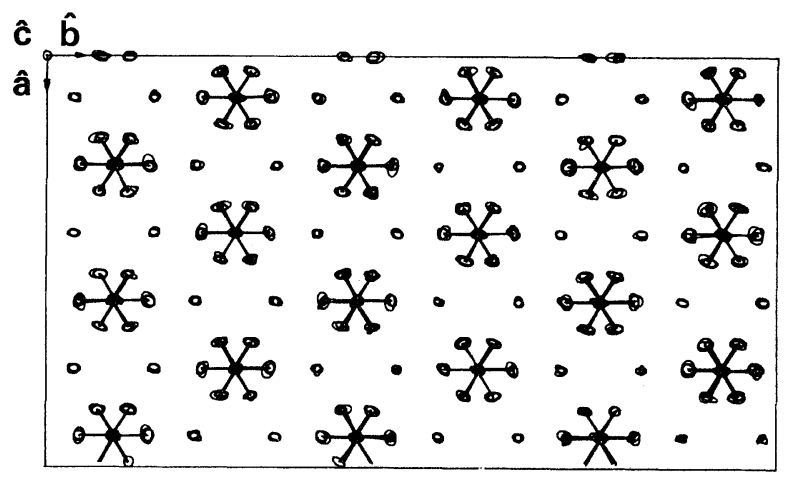

(a)

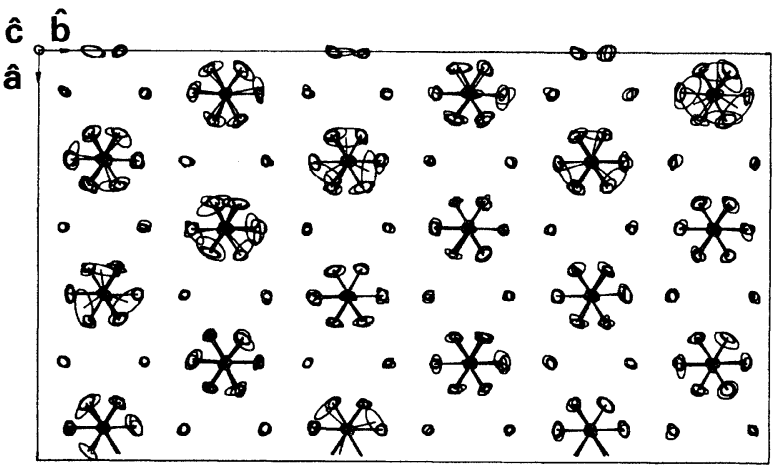

(b)

FIG. 7. Projections of the atomic positions in the $\mathrm{c}$ direction in the average structures at $T=420 \mathrm{~K}$ (a) and $T=460 \mathrm{~K}$ (b), obtained from the supercell molecular dynamics simulation. The ellipsoids centered at the atomic positions indicate the amplitudes of the thermal motions of the atoms. 
the nitrogen atoms with the nearest three oxygen atoms and the ellipses indicate the thermal motion of the atoms. There are six nitrate ions in each stack. On comparing Fig. 7(a) with Fig. 7(b) we see that some nitrate ions in the higher-temperature structure have rotated away from their orientations in the low-temperature structure. As the sample was heated to higher and higher temperatures, more and more nitrate ions rotated until they were all in a disordered state.

The experimental structure above the Pmcn phase is the disordered $\beta$ phase. ${ }^{12}$ It is very similar to the ferroelectric $R 3 m$ structure in Fig. 3, except that the nitrate ions are in dynamic disorder between two orientations separated by a $60^{\circ}$ rotation about the triad axes. Such disordering is also accompanied by the shift of the $\mathrm{K}^{+}$. ions in the c direction, and thus eliminates the spontaneous polarization in the system.

A comparison between the static Pmcn structure and the average $\beta$-phase structure indicates that the $\alpha \rightarrow \beta$ transition involves large translations of the $\mathrm{K}^{+}$and the $\mathrm{NO}_{3}{ }^{-}$ions in the $\mathbf{a}, \mathbf{b}$ plane. ${ }^{12}$ Our molecular dynamics simulation shows that the transition started out with rotations of the $\mathrm{NO}_{3}{ }^{-}$ions about $\mathbf{c}$ in the a,b plane. As will be discussed in Sec. $\mathrm{V}$, such rotations are associated with the abnormally large thermal expansion in the $c$ direction. Figure 8 shows the lattice constant $c$ obtained from our simulation as a function of temperature. As can be seen, the $c$ axis increases much faster above $440 \mathrm{~K}$, when the nitrate ions commence their rotations. Such large c-axis expansion will enlarge the spaces between the $\mathrm{K}^{+}$and the nitrate ion layers and further weaken the interactions between them, to the point that these large translations of the layers of ions become possible.

Unfortunately this component of the $\alpha \rightarrow \beta$ transition involves a radical repacking of the ions and thus lies far outside the time scales accessible to molecular dynamics. Another possible difficulty for the simulation is that the $\alpha$ and $\beta$ structures have different numbers of ions in their unit cells while in the molecular dynamics the motions of the ions are restricted by the periodic boundary conditions necessary to simulate an infinite lattice. We tried to

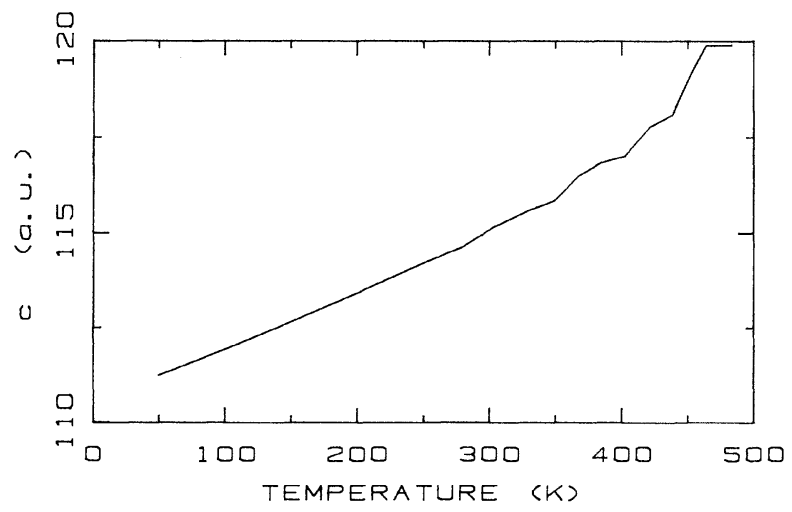

FIG. 8. The length of the c axis (atomic units) as a function of temperature from the molecular dynamics simulation of the $\alpha \rightarrow \beta$ transition. overcome these difficulties by using large supercells, redefining hexagonal axes, running at elevated temperatures and using long relaxation times, but could not produce the $\beta$ phase from the Pmcn structure. However, from the discussions above, our simulation clearly showed that the $\alpha \rightarrow \beta$ transition started at about $440 \mathrm{~K}$, which is in close agreement with the experimental value of $401 \mathrm{~K}\left(128^{\circ} \mathrm{C}\right)$. Indeed, this discrepancy may be due principally to statistical uncertainties in the molecular dynamics simulations. In Ref. 12, neutron diffraction measurements were carried out for the Pmcn structures at different temperatures and the $\mathbf{c}$ axis was found to increase by $1.28 \%$ as the temperature was changed from 25 to $100^{\circ} \mathrm{C}$. For our theoretical simulation this value was $1.36 \%$. The close agreement indicates that although the absolute values of the lattice constant $c$ were noticeably shorter than experiments $\left(5.16 \%\right.$ at $25^{\circ} \mathrm{C}$ and $5.09 \%$ at $100^{\circ} \mathrm{C}$ ), our theoretical approach gives an excellent description of the abnormally large c-axis expansion, which is a critical factor in driving the phase transitions in this system.

\section{B. The $\gamma \rightarrow \beta$ transition}

We simulated the $\gamma \rightarrow \beta$ transition with a supercell of 270 ions, formed by tripling in the $\mathbf{a}$ and $\mathbf{b}$ directions and doubling in the c direction the $R 3 \mathrm{~m}$ unit cell. Starting from the static theoretical $R 3 m$ structure obtained earlier (Fig. 4), we heated the sample in stages and allowed an equilibration time of $10 \mathrm{ps}$ at each stage. The average ion positions, lattice constants, polarization, etc., were then calculated. Since the $\gamma \rightarrow \beta$ transition is marked by the loss of the spontaneous polarization, such a polarization would be most convenient in monitoring the transition. In Fig. 9, we plotted the average spontaneous polarization collected at each stage in the heating process as a function of temperature. It shows that the spontaneous polarization started to drop very rapidly at about $150 \mathrm{~K}$ and reached near zero around $180 \mathrm{~K}$. This is definitely the $\gamma \rightarrow \beta$ transition. (We will discuss the relations between this transition temperature and the experimental value later in this section.)

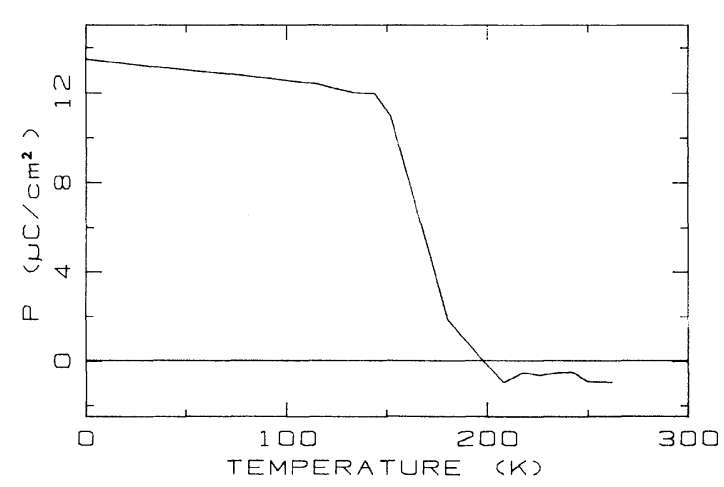

FIG. 9. Average spontaneous polarization $P$ at each temperature in the molecular dynamics simulation of the $\alpha \rightarrow \beta$ transition. 
The nonzero values of the spontaneous polarization in Fig. 9 above the transition is an artifact of the computation of the polarization. From molecular-dynamics simulation we obtained the polarization, which is just the average, over the time, of the quantity $\mathbf{p}^{\prime}=\sum_{i} q_{i} \mathbf{r}_{i}$, where the sum on $i$ runs over all the ions with charges $q_{i}$ and positions $\mathbf{r}_{i}$. However, this value of $p^{\prime}$ contains a part $p^{\prime \prime}$ that results purely from the selection of the unit cell, and is due to the fact that the $R 3 m$ unit cell has three formula units, an odd number. This part $p^{\prime \prime}$ is not the reversible spontaneous polarization and is simply the dipole moment of a pair of $\mathrm{K}^{+}$and $\mathrm{NO}_{3}{ }^{-}$ions separated by $c / 2$. To obtain the reversible spontaneous polarization $p$, one has to subtract this part from $p^{\prime}$, i.e., $p=p^{\prime}-p^{\prime \prime}$. For Fig. 8 we chose $p^{\prime \prime}=c / 2 v$, at each temperature with $c$ and $v$ being the average c-axis length and average unit cell volume, respectively. While this subtraction gives the spontaneous polarization approximately, it does not account for the statistical fluctuations in $c$ and $v$ during the molecular dynamics calculation. In particular, the fact that the $\mathrm{NO}_{3}{ }^{-}$ions are not exactly planar at elevated temperatures is not included. However, this inaccuracy should not affect the basic behavior of the spontaneous polarization as a function of temperature and certainly does not invalidate our previous discussion.

To see the structural changes at the transition, we plot-
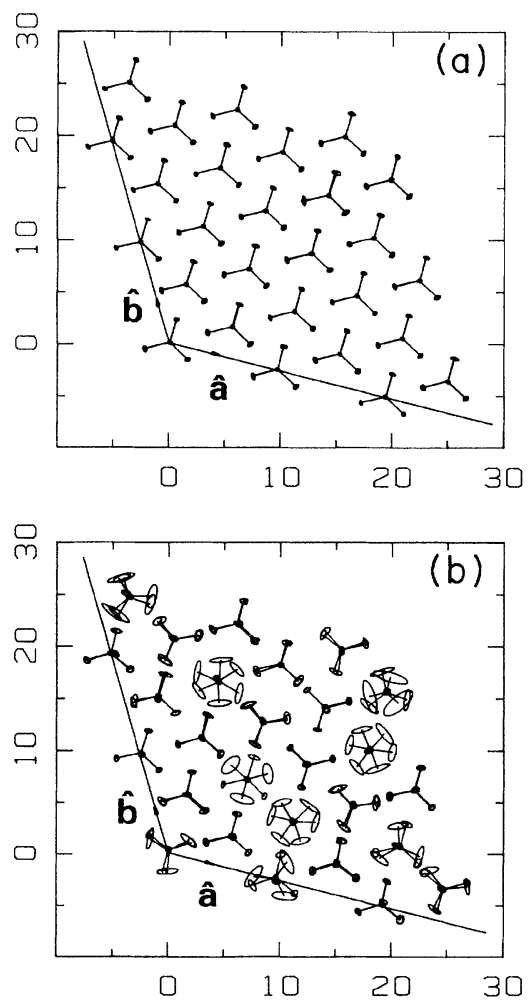

FIG. 10. Projections of the atomic positions in the direction (with the c axis pointing out of the page) in the average structures at $T=140 \mathrm{~K}$ (a) and $T=180 \mathrm{~K} \mathrm{(b)}$ in the molecular dynamics simulation of the $\gamma \rightarrow \beta$ transition. ted the projections of the average atomic positions below $(140 \mathrm{~K})$ and above $(180 \mathrm{~K})$ the transition in Figs. 10(a) and $10(\mathrm{~b})$, respectively. As before, the thermal motions of the ions are indicated by ellipses centered at the average positions. At $140 \mathrm{~K}$ all of the nitrate ions, two of them in each stack, have the same orientation. At $180 \mathrm{~K}$ about half of the nitrate ions have rotated about the $\mathbf{c}$ axis: for some stacks both of the nitrates have rotated $60^{\circ}$ from their lower-temperature orientations, and for some stacks the two nitrates occupy, respectively, the pair of orientations separated by a $60^{\circ}$ rotation about the c axis; this is just the dynamically disordered state of the $\beta$ phase of $\mathrm{KNO}_{3}$.

The interesting feature of the $\gamma \rightarrow \beta$ transition is the anisotropic thermal expansion. It was found experimentally $^{21}$ that below the transition, the thermal expansion along the $\mathrm{c}$ axes is $\sim 2 \times 10^{-4} \mathrm{~K}^{-1}$, about one order of magnitude larger than that along $a$. At the transition itself, the c axis increases discontinuously from 9.14 to 9.64 $\AA$, a 5.2\% change, and the a axis decreases from 5.48 to $5.42 \AA, a-1.1 \%$ change. In Fig. 11 we show the average lattice constants $a$ and $c$ from our simulation as functions of temperature. The anisotropic behavior of the lattice constants is well reproduced. Across the transition from 140 to $180 \mathrm{~K}$ the c axis increased by $8.9 \%$ and the a axis decreased $1.9 \%$. Both numbers are larger than the discontinuous change observed in experiment, which is not surprising since they include both the abrupt changes and the gradual changes before and after the transition. This shows that our theoretical description agrees with experiment very well as far as these general characteristics of the transition are concerned.

\section{The $\gamma \rightarrow \alpha$ and $\beta \rightarrow \gamma$ transitions}

In Sec. III we have shown by static relaxations with and without symmetry constraints that the $R 3 m$ structure is metastable since it is fully relaxed, i.e., the forces and stresses are zero, but it has a potential energy higher

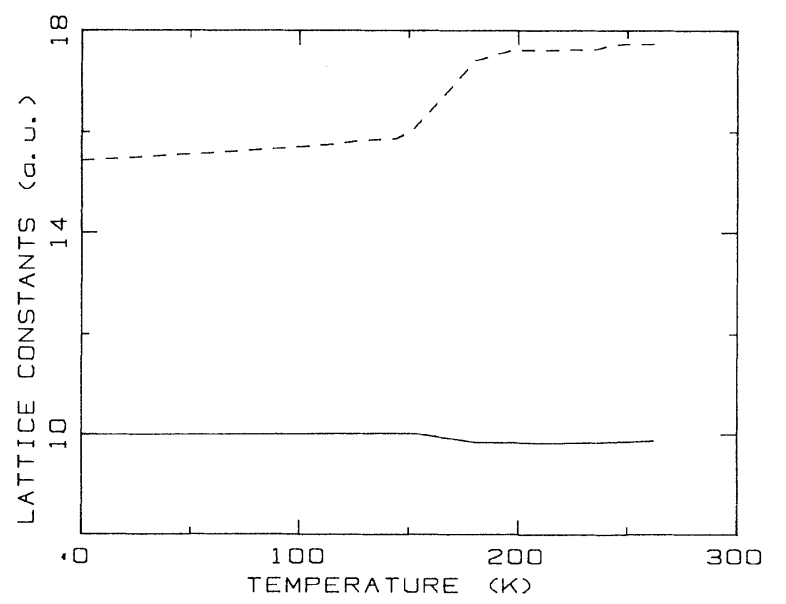

FIG. 11. Average lattice constants a (solid line) and c (dashed line) as functions of temperature in the simulation of the $\gamma \rightarrow \beta$ transition. 
than that for the Pmcn structure by $88 \mathrm{meV}$ per formula unit. This metastability was confirmed by molecular dynamics relaxations. Starting from the $R 3 \mathrm{~m}$ structure we tried supercell dynamical relaxation by various means: large number of ions, long relaxation time (within the limit of molecular dynamics simulations), large initial kinetic energy, and defining the structure by orthorhombic lattices. However, the structure did not show any indications of the $\gamma \rightarrow \alpha$ transition such as the translation of the $\mathrm{K}^{+}$ions away from their positions exactly above and beneath the $\mathrm{N}$ atoms in the $R 3 m$ structure.

This metastability of the $R 3 m$ structure is observed experimentally. It was shown ${ }^{11}$ that if the crystal is kept dry and in a vacuum, and cooled fairly rapidly, the $\gamma$ phase can be cooled down to room temperature, much lower than the normal $\gamma \rightarrow \alpha$ transition temperature $115^{\circ} \mathrm{C}$, and kept there metastably; it can be reheated to $120^{\circ} \mathrm{C}$ without reverting to the $\alpha$ phase.

This indicates that the $\gamma \rightarrow \alpha$ transition can occur only within a particular temperature range, unless it is induced by defect surfaces. If this is the case, it should be impossible to simulate via molecular dynamics which treats only perfect crystals and has large statistical uncertainty in the temperature due to the limited size of the supercell. Moreover, a process that depends so sensitively on cooling is too slow to be simulated by molecular dynamics. A similar situation occurs as we tried to simulate the $\beta \rightarrow \gamma$ transition by quenching the $\beta$ structure [Fig. 10(b)]. The equilibration time that allowed molecular dynamics is simply too short to allow the nitrate ions to adjust their orientations so that they do not get locked into other metastable configurations.

These considerations show that the transition temperature we have obtained in simulating the $\gamma \rightarrow \beta$ transition are not the actual values but merely measure the kinetic energy required for a perfect $R 3 \mathrm{~m}$ structure to transform, and therefore should not be compared directly with the experimental transition temperatures. However, the characters revealed in the simulation clearly match those found in experiment and thus should still be considered as a valid description for the actual phase transition.

\section{QUASIHARMONIC LATTICE-DYNAMICS CALCULATIONS AND FOURIER ANALYSIS OF THE ROTATIONAL MOTION OF $\mathrm{NO}_{3}^{-}$}

We have shown that both the $\alpha \rightarrow \beta$ and $\gamma \rightarrow \beta$ transitions were initiated by rotations of the nitrate ions about the $\mathbf{c}$ axis and were accompanied by abnormally large thermal expansion in the c direction. We now show that these two phenomena are closely interconnected and result from the very anharmonic nature of the lattice motion, particularly in the transition region.

A simple and direct way to illustrate this point is to perform lattice dynamics calculations for the static but thermally expanded structure at different temperatures and examine the changes of normal mode frequencies due to this expansion, whose principal component is along the c direction. From the supercell molecular dynamics calculations presented in Sec. IV A, we obtained the average lattice constants at various temperatures, which give the sizes of the thermally expanded structures. If we use these lattice constants for the static structure of $\mathrm{KNO}_{3}$ in the ordered phase, i.e., the Pmcn structure, and then perform a static relaxation of the structure, but with the lattice constants fixed, we can obtain relaxed structures that should reproduce the dynamical properties of the lattice structures at the various temperatures. Lattice-dynamics calculations for these relaxed structures will then give normal mode frequencies as functions of temperature, which should reveal the connection between the dynamics of the system and the anomalous thermal expansion.

It should be pointed out that these calculations were not exactly the quasiharmonic calculations in the usual sense, since the static structures were not fitted to experiment. In particular, the lattice constants in the theoretical structures were all shorter by about $5 \%$ as pointed out earlier. Therefore the frequencies obtained here cannot be directly compared with experiment. However, their variation as functions of temperature should correctly reflect the anharmonic behavior, since the theoretical potentials do indeed simulate the large thermal expansion in the system.

Since our focus is mainly on the rotation of the nitrate ions, we limit our calculations to the zone-center normal-mode frequencies. In Fig. 12, we show the frequencies of the lowest six modes for Pmcn structures of $\mathrm{KNO}_{3}$ at temperatures from $0 \mathrm{~K}$ (for the static relaxed structure given in Fig. 12) up to $600 \mathrm{~K}$.

Figure 12 shows that when temperature increases and the lattice expands, mainly in the c direction, the frequencies of all of the modes decrease, except that of the $A_{\mathrm{g}}$ mode. However, the mode whose frequency decreases most rapidly is the $A_{u}$ mode, which becomes the

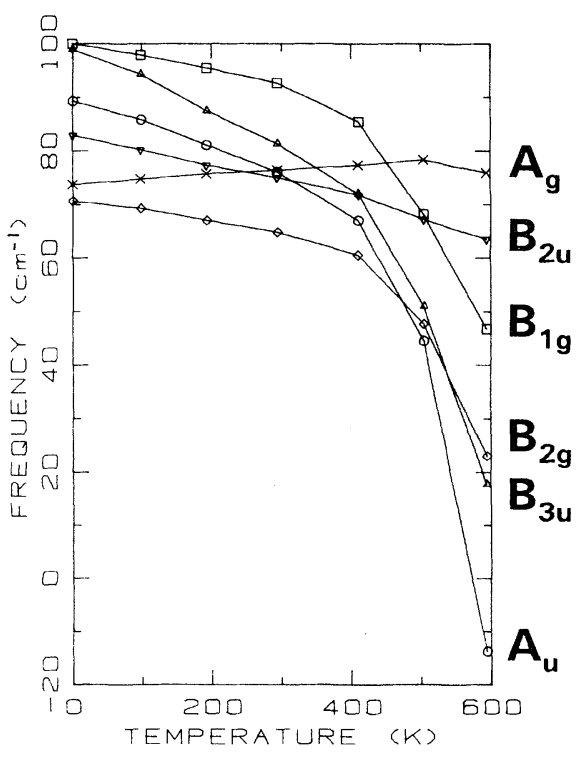

FIG. 12. Normal mode frequencies as functions of temperature for the lowest $A_{g}(\times), B_{2 u}(\triangle) B_{1 g}(\square), B_{2 g},(\diamond), B_{3 u}(\nabla)$, and $A_{u}(\mathrm{O})$ modes of the Pmcn structures of $\mathrm{KNO}_{3}$. 
lowest mode at about the theoretical transition temperature and unstable with an imaginary frequency when temperature is increased further. An examination of the normal-mode pattern for this $A_{u}$ mode shows that it involves purely rotation of the nitrate ions. At $T=0 \mathrm{~K}$, the rotation is mainly about the $\mathbf{c}$ axis but also about the $\mathbf{b}$ axis with a much smaller amplitude. As temperature increases the $b$ axis component becomes smaller and smaller, and finally vanishes at about $400 \mathrm{~K}$ and above, so that the rotation becomes purely one about the c axis. Figure 13 illustrates the normal-mode pattern for the $A_{u}$ mode at about $500 \mathrm{~K}$. This shows that the rotations of the nitrate ions that initiate and drive the transition, as we have seen in the molecular dynamics simulation, are directly associated with the softening of this $A_{u}$ mode.

The softening of the rotational mode of the nitrate about the $c$ axis can be understood by considering the interionic interactions in the Pmcn structure of $\mathrm{KNO}_{3}$. A close examination of the Pmcn structure (Fig. 2) will show that there are three $\mathrm{K}^{+}$ions just above any nitrate ion and three just below. Apart from the other atoms in the same nitrate ion, the closest neighbors for an oxygen atom are these $\mathrm{K}^{+}$ions. The interactions between these and the oxygen atom restrict the rotation of the nitrate ions. As the c axis expands substantially, the distances between the layers of $\mathrm{K}^{+}$ions and layers of nitrate ions becomes larger, and the restriction on the rotations of the nitrate ions become weaker, and thus the frequency for the rotational motion becomes smaller, and eventually becomes imaginary.

As a consequence, the rotational motion of the nitrate ions about the c axis is strongly anharmonic: a result of the loose packing of the Pmcn structure in the c direction. Because of this loose binding the structure expands rapidly in this direction as the temperature is raised, permitting greater libration of the nitrate ions. In turn, these librations of the nitrates further enhance the expansion of lattice in the c direction. At the transition temperature, these librations become nearly free rotations and the transition takes place.

In Fig. 12, the $B_{3 u}, B_{2 g}$, and $B_{1 g}$ modes also show substantial softening at the transition. At $T=0 \mathrm{~K}$, the $B_{3 u}$

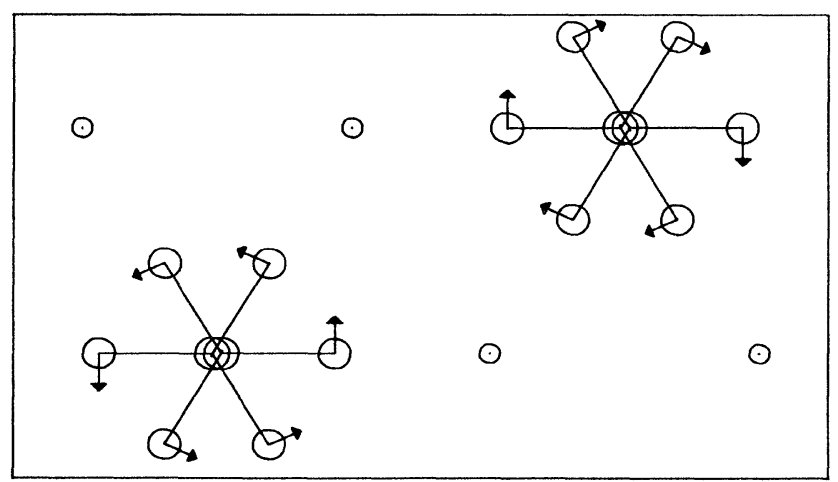

FIG. 13. Projection of the normal mode pattern for the softening $A_{u}$ mode at $500 \mathrm{~K}$ in the c direction. All of the displacements are in the a,b plane. mode consists of the rotations of the nitrate ions about the $b$ axis and a slight rotation about the $c$ axis. As the temperature increases the $\mathbf{b}$ component of the rotation becomes smaller, while the component becomes larger, very similar to the situation for the $A_{u}$ mode. However, the b component does not vanish even at $600 \mathrm{~K}$. At $T=0$, the $B_{2 g}$ mode involves translations of the nitrate and the $\mathrm{K}^{+}$ions in the a,b plane. As the temperature increases, it evolves into rotations of the nitrate ions about the c axis, but with the axis of rotation through one of the oxygens. At $T=0 \mathrm{~K}$, the $B_{1 g}$ mode involves the translation of the nitrate ions in the $\mathbf{a}, \mathbf{b}$ plane, but becomes pure rotation of the nitrate ions about the $c$ axis for temperatures higher that $300 \mathrm{~K}$. Obviously the softening of these three modes is due to the same cause as that of the $A_{u}$ mode, i.e., the large c-axis expansion, and they certainly contribute to the destabilization of the lattice at the transition. However, the dominating instability in the structure is the softening of the $A_{u}$ mode.

A point worth mentioning is that the $A_{u}$ mode in the Pmcn structure is a "silent" mode, meaning it is neither Raman nor infrared active. Therefore its softening upon the transition cannot be measured by Raman or infrared experiment. However, as we have seen in Fig. 12, other modes also have substantial softening and could certainly be observed either in Raman or infrared.

Similarly, we calculated the normal mode frequencies for the $\gamma$-phase structures at $T=0 \mathrm{~K}$, and at $T=150 \mathrm{~K}$, just before the transition seen in molecular dynamics simulation. At $T=0 \mathrm{~K}$, the lowest frequency modes involving rotations of the nitrate ions are an $A_{2}$ mode at $100 \mathrm{~cm}^{-1}$ (also a "silent" mode), and an $E$ mode at 78 $\mathrm{cm}^{-1}$, which is also the lowest optical mode. At $T=150$ $K$, the frequencies of these modes are reduced drastically to 68 and $45 \mathrm{~cm}^{-1}$, respectively, and are now the lowest two optical modes. Despite the distinctive difference between the $R 3 m$ and the Pmcn structures, the essential features are still the same, as far as the rotational motion of the nitrate ions are concerned. The $R 3 \mathrm{~m}$ structure also contains the $\mathrm{K}^{+}$ion layers that separate the nitrate ions layers and similarly the closest neighbors for an oxygen atom are these $\mathrm{K}^{+}$ions, besides the other atoms in the same nitrate. Therefore, the rotation of the nitrates about the $\mathbf{c}$ axis is also hindered by $\mathrm{K}^{+}$ion layers and when these layers move further apart as the lattice expands markedly in the c direction, the rotation becomes nearly free, and so initiates the order-disorder transition.

The softening of the rotational motion of the nitrate ions, revealed by the quasiharmonic calculation above, can also be seen, perhaps more directly, by performing Fourier transform calculations for the time evolution of these rotational motions. From our molecular dynamics simulation of the Pmcn structure, we can obtain the function $\varphi(t)$, which is the angle between the instantaneous orientations of a nitrate ion and its orientation in the average structure. Then by calculating the Fourier transform of $\varphi(t)$, i.e., $\varphi(\omega)$, and taking the average over all the nitrates in the supercell, we obtain an average frequency spectrum $\overline{|\varphi(\omega)|}$ for the rotational motions of the nitrate ions.

The results are shown in Fig. 14 for several tempera- 


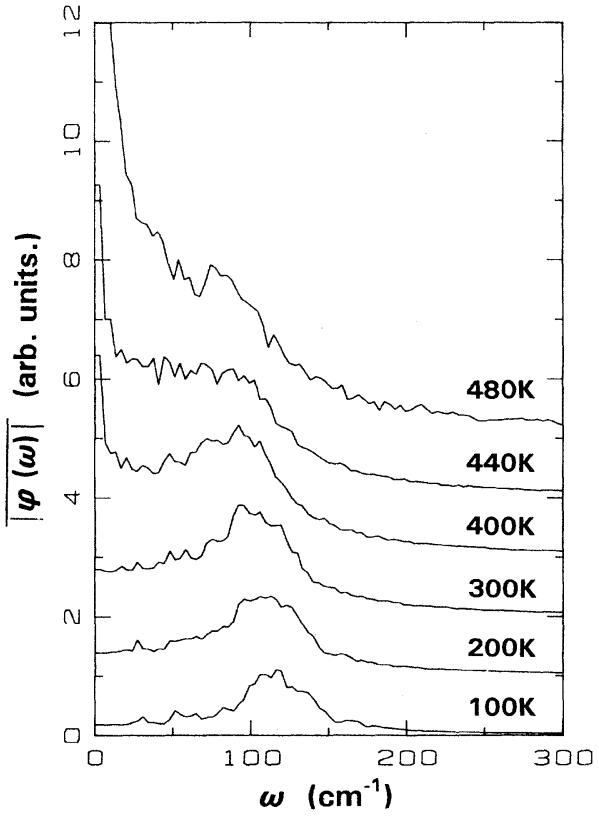

FIG. 14. Average frequency spectra $\overline{\varphi(\omega) \mid}$ for the rotational motions of the nitrate ions.

tures and are extremely interesting. The rotational motions of the nitrate ions clearly have two components: a quasiharmonic response with a well-defined average frequency, which actually softens in a manner that closely parallels that of the quasiharmonic normal modes in Fig. 12 and clearly does not approach zero at the transition, and a "central peak," which appears close to and below $T_{c}(\sim 440 \mathrm{~K})$ and becomes very large at and above $T_{c}$. This behavior is a very natural consequence of the transition dynamics of the system. At low temperatures the $\mathrm{NO}_{3}{ }^{-}$ions are well localized orientationally and librating in the wells of a rotationally periodic potential. As the temperature rises the librational amplitudes increase both due to the increase in kinetic energy and the large $c$ axis expansion which makes the wells shallower, hence the downshift in frequency of the (still basically harmonic) motion. Then as temperature approaches $T_{c}$, the ions begin to reorient between wells; this introduces major jumps in rotational angles that produce the central peak in the transform. However even at $T_{c}$ or higher temperature, the potential wells are still present and within them the $\mathrm{NO}_{3}{ }^{-}$ions are still undergoing quasiharmonic librations for several cycles between jumps.

\section{CONCLUSIONS}

Through static structural relaxations and molecular dynamics simulations, we have developed an $a b$ initio theoretical description for both the $\alpha$ and $\gamma$ ordered phases of $\mathrm{KNO}_{3}$ and their transitions to the highertemperature disordered $\beta$ structure. Our results are in close agreement with experiment for the static structures and, more importantly, display the abnormally large thermal expansion observed for the $c$ direction. We have shown that both the $\alpha \rightarrow \beta$ and $\gamma \rightarrow \beta$ transitions are initiated by the nearly free rotations of the nitrate ions about the $\mathbf{c}$ axis. By performing lattice-dynamical calculations in the spirit of quasiharmonic approximation, we showed that the rotation of the nitrate ions and the large c-axis thermal expansion are closely interconnected and are results of the strong anharmonic characters of these motions. This anharmonicity is attributed to the loose packing in both the $\alpha$ and $\gamma$ phases of $\mathrm{KNO}_{3}$ in the c direction and is the basic driving mechanism for the transition.

\section{ACKNOWLEDGMENTS}

This work was supported by the U.S. Army Research Office. We should also like to acknowledge the Pittsburgh Supercomputing Center for the support of the CPU intensive computations.
${ }^{1}$ H. M. Lu and J. R. Hardy, Phys. Rev. Lett. 64, 661 (1990).

${ }^{2}$ H. M. Lu and J. R. Hardy, Phys. Rev. B 42, 8339 (1990).

${ }^{3}$ R. G. Gordon and Y. S. Kim, J. Chem. Phys. 56, 3122 (1971).

${ }^{4}$ L. L. Boyer, Phys. Rev. Lett. 42, 584 (1979). A fuller account is given in Phys. Rev. B 23, 3673 (1981).

${ }^{5}$ L. L. Boyer, Phys. Rev. Lett. 45, 1858 (1980); 46, 1172 (1981).

${ }^{6}$ L. L. Boyer and J. R. Hardy, Phys. Rev. B 24, 2527 (1981).

${ }^{7}$ L. L. Boyer, J. Phys. C 17, 1825 (1984).

${ }^{8}$ V. Katkanant, P. J. Edwardson, J. R. Hardy, and L. L. Boyer, Phys. Rev. Lett. 57, 2033 (1986).

${ }^{9}$ P. J. Edwardson, V. Katkanant, J. R. Hardy, and L. L. Boyer, Phys. Rev. B 35, 8479 (1987).

${ }^{10}$ V. Katkanant, P. J. Edwardson, and J. R. Hardy, Phase Transitions 15, 103 (1989).

${ }^{11}$ H. D. Megaw, Crystal Structures: A Working Approach (Saunders, Philadelphia, 1973), p. 493.

12J. K. Nimmo and B. W. Lucas, J. Phys. C 6, 201 (1973); Acta
Crystallogr. B32, 1968 (1976).

${ }^{13}$ S. Sawada, S. Nomura, and Y. Asao, J. Phys. Soc. Jpn. 13, 1549 (1958).

${ }^{14}$ S. V. Karpov and A. A. Shultin, J. Phys. Chem. Solids 29, 475 (1968).

${ }^{15}$ A. A. Shultin and S. V. Karpov, J. Phys. Chem. Solids 30, 1981 (1969).

${ }^{16}$ M. B. Brooker, Can. J. Chem. 55, 1242 (1977).

${ }^{17}$ E. Clementi and C. Roetti, At. Data Nucl. Data Tables 14, 177 (1974).

${ }^{18}$ R. S. Mulliken, J. Chem. Phys. 23, 1833 (1955).

${ }^{19}$ M. J. Frisch et al., GAUSSIAN86 (Carnegie-Mellon Quantum Chemistry Publishing Unit, Pittsburgh, PA, 1984).

${ }^{20}$ D. Liu, H. M. Lu, F. G. Ullman, and J. R. Hardy, Phys. Rev. B 44, 7387 (1991).

${ }^{21}$ M. Kantola and T. Tarna, Ann. Acad. Sci. Fenn. A, VI, No. 335 (1970). 